\title{
HEPATECTOMIA POR VIDEOLAPAROSCOPIA. Realidade?
}

\author{
Luiz A. Carneiro D'ALBUQUERQUE e Paulo HERMAN
}

\begin{abstract}
RESUMO - Racional - Apesar do grande avanço nos últimos anos, a ressecção hepática é uma das últimas fronteiras a serem vencidas pela cirurgia laparoscópica. As publicações na literatura mundial referem-se a relatos de casos ou avaliação de pequenas séries de pacientes que são vistas com ceticismo por muitos cirurgiões. Trata-se de procedimento difícil e longo para o qual são necessários experiência em cirurgia hepática e laparoscópica, além de equipamento adequado. Foram avaliadas as indicações e os aspectos técnicos, realizando-se análise crítica do método. Conclusão - Analisando-se os resultados de artigos publicados, pode-se concluir que a hepatectomia por videolaparoscopia é hoje operação segura e factível e portanto, uma realidade, tendo no entanto, indicação para casos selecionados. A ressecção hepática por laparoscopia deve fazer parte do armamentário terapêutico do cirurgião de fígado.
\end{abstract}

DESCRITORES - Hepatectomia. Laparoscopia.

\section{INTRODUÇÃO}

Os avanços técnicos da laparoscopia revolucionaram a terapêutica cirúrgica de grande número de doenças. Nos últimos anos, esses avanços têm possibilitado a ressecção de órgãos sólidos como adrenal, rim e baço. No entanto, algumas cirurgias como a ressecção hepática, publicadas na literatura mundial como descrição de casos ou séries pequenas, ainda são vistas com ceticismo.

A ressecção hepática é uma das últimas fronteiras a serem vencidas pela cirurgia laparoscópica. As razões para o limitado desenvolvimento da hepatectomia laparoscópica não são muito claras, visto que a ressecção hepática é procedimento direto e único onde não há necessidade de reconstrução ou anastomoses. Por outro lado, manobras quase sempre necessárias durante a cirurgia do fígado, como a sua ampla mobilização e exposição, controle dos pedículos vasculares, palpação minuciosa e ultra-sonografia intra-operatória parecem difíceis de serem realizados através de videolaparoscopia (VL). Além disso, fatores como a transecção do parênquima, o potencial de hemorragia intra-operatória e o risco de embolia gasosa tornam os procedimentos laparoscópicos tema controverso.

Discute-se muito, também, se esse procedimento deve ser realizado por cirurgiões com experiência em cirurgia laparoscópica ou por aqueles com experiência em cirurgia hepática. Não há dúvida de que essas duas qualificações são absolutamente necessárias e recomenda-se que a cirurgia deva ser realizada, ao menos no início da experiência, por equipe da qual participem um cirurgião com experiência em cirurgia do fígado e outro com experiência em cirurgia videolaparoscópica.

Algumas equipes formadas por cirurgiões treinados em ambas têm realizado ressecções hepáticas por $\mathrm{VL}^{(4,6,16,19,31)}$, no entanto esse procedimento ainda não foi adequadamente avaliado e discutido.

Serão abordados, a seguir, as indicações, os aspectos técnicos e será feita uma análise crítica desse método de tratamento tão em voga nos dias de hoje.

\section{INDICAÇÕES}

A maior parte dos estudos refere-se à ressecção de tumores benignos ou ao tratamento de cistos hepáticos. Os melhores candidatos são pacientes jovens com tumores benignos superficiais ou periféricos, com indicação de ressecção limitada do parênquima. Recomenda-se que o método deva ser empregado nas ressecções das porções ântero-laterais do fígado (segmentos II, III, IVb, V, VI) ou ainda na porção esquerda do lobo caudado.

Dentre as indicações para lesões benignas, podemse destacar: cistos hepáticos simples, cisto hidático, cistoadenoma biliar hepático, hemangioma hepático gigante e adenoma hepático.

Dentre essas indicações, a doença cística é melhor tratada por destelhamento ("unroofing"), isto é, a ressecção da cúpula do cisto, por videolaparoscopia e não por ressecção. Em relação ao cisto hidático, existem relatos favoráveis à aspiração e injeção de agentes escolicidas por via laparoscópica, mas essa conduta não está bem estabelecida $^{(1)}$. O cistoadenoma biliar, por se tratar de

Departamento de Gastroenterologia da Faculdade de Medicina da Universidade de São Paulo, São Paulo, SP.

Endereço para correspondência: Dr. Luiz A. Carneiro D’Albuquerque - Rua Evaristo de Moraes, 55 - apt.111 - 04007-070 - São Paulo, SP E-mail: lamtca@terra.com.br 
lesão pré-neoplásica, tem indicação de ressecção e a laparoscopia pode ser boa opção. $\mathrm{O}$ hemangioma hepático raramente tem indicação cirúrgica, mas casos com grandes tumores de lobo esquerdo com compressão gástrica, ocasionando sintomas digestivos, podem ser boa indicação. A melhor indicação é, sem dúvida, o adenoma hepático, que geralmente acomete mulheres jovens e, pelo seu potencial de malignização e de hemorragia, deve ser ressecado.

Deve-se ressaltar que a cirurgia laparoscópica não deve ampliar as indicações cirúrgicas nos tumores benignos do fígado. As indicações devem ser as mesmas que para a cirurgia convencional.

Têm sido discutidas as indicações para o tratamento das metástases hepáticas e do hepatocarcinoma em pacientes com boa reserva funcional hepática, onde o papel da laparoscopia ainda não está bem estabelecido. A possibilidade de disseminação tumoral e a manutenção de margens adequadas, além da ausência de trabalhos mostrando resultados a longo prazo, são potenciais desvantagens do método.

Há pouquíssimos estudos na literatura mostrando experiência no tratamento laparoscópico de metástases hepáticas como o de O'ROURKE et al. ${ }^{(27)}$, na Austrália, que relataram 28 hepatectomias por videolaparoscopia para metástases hepáticas com bons resultados. No entanto, os resultados ainda são iniciais para uma análise mais detalhada.

Recentemente, vários autores, especialmente do leste asiático ${ }^{(17}$ ${ }^{18,20)}$, têm proposto a ressecção de pequenos hepatocarcinomas superficiais em segmentos ântero-laterais do fígado, com bons resultados. LAURENT et al. ${ }^{(22)}$ e CHERQUI et al. ${ }^{\left({ }^{()}\right.}$, na França, demonstraram que a hepatectomia por VL reduziu o sangramento, a morbidade e a mortalidade de pacientes cirróticos. Da mesma forma, BELLI et al. ${ }^{(3)}$, na Itália, também têm mostrado entusiasmo na ressecção de hepatocarcinomas superficiais ou exofíticos em pacientes com cirrose hepática compensada. Baseado nesses bons resultados iniciais e, pela ocorrência de menos aderências quando de uma reoperação como o transplante de fígado, talvez no futuro, este seja o procedimento de escolha para o pequeno hepatocarcinoma superficial.

\section{Aspectos técnicos}

A hepatectomia por VL é um procedimento difícil, trabalhoso e longo para o qual é necessária experiência em cirurgia hepática e laparoscópica, paciência e a disponibilidade de equipamento adequado.

A padronização técnica, com o uso de tecnologia já existente para a cirurgia convencional e adaptada para a laparoscopia é, segundo BUELL et al. ${ }^{(5)}$, ponto importante para facilitar o procedimento. Os avanços tecnológicos nos últimos anos propiciaram a realização da hepatectomia laparoscópica. Dentre os novos equipamentos disponíveis, devem-se destacar: transdutores de ultra-sonografia laparoscópica, grampeadores vasculares para laparoscopia, aparatos para secção do parênquima como bisturi harmônico, tissuelink e liga-sure para laparoscopia.

Outra técnica de grande utilidade, especialmente no início da experiência, é a utilização de equipamento com auxílio da mão ("hand assisted"), que consiste em um anel colocado sobre uma pequena incisão na pele de cerca de $7 \mathrm{~cm}$ ligado a um saco plástico, onde se pode introduzir a mão na cavidade abdominal sem que haja vazamento de gás do pneumoperitônio. FONG et al. ${ }^{(13)}$ reforçam que com o uso desta tecnologia, o procedimento se torna mais fácil e seguro, além de aumentar a confiança na obtenção das margens e facilitar a remoção do espécime.

O controle vascular é uma grande preocupação nas hepatectomias, especialmente na ressecção de tumores próximos a grandes vasos ou nas ressecções maiores. O pinçamento do pedículo hepático (manobra de Pringle) pode ser realizado com facilidade, colocandose um cadarço ao redor do hilo hepático, confeccionando-se um torniquete para o controle do fluxo hepático. O clampeamento do pedículo hepático também é possível através do uso de pinças vasculares adaptadas para a videolaparoscopia. Recentemente, em nosso meio, MACHADO et al. ${ }^{(25,26)}$ publicaram técnica onde é possível se obter o controle do pedículo de apenas um dos lobos do fígado (hemi-Pringle), propiciando ressecção segura, sem levar à isquemia do lobo contra-lateral. Alguns grupos utilizam grampeadores mecânicos para facilitar as ressecções, principalmente dos segmentos II e III ${ }^{(24)}$.

A escola cirúrgica francesa, pioneira em ressecções anatômicas hepáticas, também vem trazendo contribuições em ressecções hepáticas por videolaparoscopia. VIBERT et al. ${ }^{(33)}$ publicaram recentemente uma série de 84 hepatectomias por VL, sendo 46 ressecções anatômicas e, em 26 pacientes, mais de três segmentos hepáticos foram ressecados. Neste estudo onde a ultra-sonografia intra-operatória foi realizada de forma rotineira, os pedículos hepáticos foram dissecados e ligados e a ressecção foi realizada de forma regrada, com resultados superponíveis aos da cirurgia convencional.

Outra preocupação é a possibilidade de se semear células tumorais quando da retirada do espécime. A peça cirúrgica deve sempre ser introduzida em saco de material resistente e sua retirada deve ser feita através da incisão umbilical, quando menor que $3 \mathrm{~cm}$, ou por incisão de apendicectomia (quando previamente presente) ou supra-púbica, nas peças maiores.

\section{DISCUSSÃO}

GAGNER et al. ${ }^{(14)}$, em 1992, publicaram a primeira hepatectomia laparoscópica para a ressecção de um tumor hepático de $6 \mathrm{~cm}$ (hiperplasia nodular focal); em 1995, FERZLI et al. ${ }^{(12)}$ reportaram a ressecção de um adenoma hepático de $9 \mathrm{~cm}$ no segmento IV. A primeira hepatectomia anatômica regrada por VL foi publicada em 1996 por AZAGRA et al. ${ }^{(2)}$ que realizaram uma bi-segmentectomia II-III em paciente com adenoma hepático. Como se pode ver, a ressecção hepática por VL é procedimento muito recente mas que tem sido utilizado com maior freqüência nos últimos anos.

Numa análise crítica da hepatectomia laparoscópica, devese levar em conta a sua factibilidade, segurança e eficácia em comparação com os procedimentos convencionais. Também devem ser avaliados os resultados a curto e longo prazo.

A factibilidade do procedimento é o critério mais avaliado na literatura e está estabelecido por equipes especialistas que é semelhante à cirurgia aberta ${ }^{(2,6,9,16,19)}$. No entanto, recomendase que o método deva ser limitado a ressecções das porções ântero-laterais do fígado (segmentos II, III, IVb, V, VI) ou ainda a porção esquerda do lobo caudado. Na sua maioria são descritas 
ressecções limitadas (no máximo dois segmentos hepáticos) para tumores menores que $5 \mathrm{~cm}$, no entanto, a localização (ânterolateral) é mais importante que o tamanho do tumor. Dentro desses critérios, os resultados são encorajadores com índices mínimos de morbidade e ausência de complicações como hemorragia ou embolia gasosa.

Em estudo multicêntrico europeu avaliando a experiência inicial com o método, DESCOTTES et al. ${ }^{(9)}$ analisaram 87 pacientes submetidos a ressecção hepática por VL e encontraram: 10\% de conversões, sendo $45 \%$ por sangramento; $6 \%$ de necessidade de transfusão de glóbulos; 5\% complicações; ausência de mortalidade. Esses resultados foram encorajadores e em outro trabalho caso-controle com 20 pacientes submetidos a ressecção convencional e 18 a laparoscópica, LESURTEL et al. ${ }^{(23)}$ observaram na cirurgia por VL: maior tempo de cirurgia e de clampeamento do pedículo hepático, porém sem repercussões clínicas; menor perda de sangue; ausência de complicações pós-operatórias além de taxas de sobrevida e de recidiva tumoral iguais à cirurgia convencional. A Tabela 1 demonstra os resultados de alguns grupos com a ressecção hepática por VL. O entusiasmo com o método vem crescendo progressivamente e as discussões sobre indicações vêm sendo ampliadas.

Não há na literatura nenhum estudo prospectivo e randomizado comparando a ressecção hepática convencional com a laparoscópica; no entanto, alguns pontos que geralmente favorecem a laparoscopia em outros procedimentos como a diminuição ou ausência de dor no pós-operatório, diminuição do tempo de internação, retornop mais rápido às atividades, preservação da parede abdominal, diminuição de aderências peritoniais e preservação da resposta imune, também devem ser utilizados como argumentos para a realização da hepatectomia por $\mathrm{VL}^{(6,14,31,33)}$. Deve-se levar em conta que na hepatectomia laparoscópica, uma pequena incisão é necessária para a retirada do espécime, porém bem menor do que a necessária para uma hepatectomia convencional. Alguns estudos demonstraram que apesar de mais longa, a hepatectomia por VL reduziu o sangramento e a morbimortalidade, especialmente em pacientes cirróticos $^{(17)}$. CHERQUI ${ }^{(8)}$ e LAURENT et al. ${ }^{(22)}$ chamam a atenção para a menor incidência de ascite, a mais freqüente complicação pós-operatória nos cirróticos, após a VL.

Não restam dúvidas de que para o tratamento dos tumores benignos, especialmente o adenoma hepático que acomete mulheres jovens, a hepatectomia videolaparoscópica tem papel importante. A grande discussão deve ser reservada para os casos de neoplasia em que a laparoscopia poderia aumentar o risco de implantação de células neoplásicas, fato não demonstrado

TABELA 1 - Resultados da hepatectomia por videolaparoscopia

\begin{tabular}{lccccc}
\hline Autor & $\mathbf{n}$ & $\begin{array}{c}\text { Benignos/ } \\
\text { malignos }\end{array}$ & Conversão & Complicação & Mortalidade \\
\hline $\begin{array}{l}\text { Descottes et } \\
\text { al." } 2003^{(9)}\end{array}$ & 87 & $87 / 0$ & $10 \%$ & $5 \%$ & 0 \\
$\begin{array}{l}\text { Gigot et al. } \\
\text { 2002, }\end{array}$ & 37 & $0 / 37$ & $13,5 \%$ & $22 \%$ & 0 \\
$\begin{array}{l}\text { Cherqui et al., } \\
2000^{(6)}\end{array}$ & 30 & $18 / 12$ & $6,6 \%$ & $20 \%$ & 0 \\
\hline *Estudos multicêntricos & & & & \\
\end{tabular}

v. $43-$ no. 3 -jul./set. 2006

Arq Gastroenterol

245 nem experimentalmente, nem em outras neoplasias como as colorretais $^{(21,30)}$.

Em estudo multicêntrico europeu, GIGOT et al. ${ }^{(15)}$ demonstraram a utilidade da ressecção hepática por laparoscopia no tratamento de tumores malignos, com índices de sobrevida em 2 anos, semelhantes à ressecção convencional. Resultados semelhantes foram encontrados por CHERQUI ${ }^{(8)}$ e LAURENT et al. ${ }^{(22)}$.

Outro ponto importante na cirurgia oncológica é a preservação de margens cirúrgicas adequadas, muitas vezes não alcançadas pela laparoscopia pela impossibilidade de palpação e falta de equipamento adequado como o uso de transdutores de ultrasonografia por VL. Não há até o momento trabalhos mostrando resultados a longo prazo da ressecção laparoscópica de neoplasias hepáticas. No entanto, sabe-se hoje que margens inferiores a 1 $\mathrm{cm}$, porém com a ressecção completa do tumor, não têm impacto negativo na sobrevida ${ }^{(10,29)}$.

A embolia gasosa secundária ao pneumoperitônio é muito rara e não foi relatada nas grandes séries de hepatectomias por $\mathrm{VL}^{(11)}$. Além disto, o desenvolvimento de retratores de parede abdominal sem o uso do gás, já foi descrito e seus benefícios demonstrados em grandes ressecções hepáticas ${ }^{(16)}$.

A hemorragia representa a maior complicação e o grande desafio no intra-operatório. A maior parte das conversões (mais de $70 \%$ ) se dá pelo sangramento intra-operatório. A seleção adequada dos pacientes, a técnica meticulosa e as técnicas de clampeamento do pedículo hepático podem reduzir essa temida complicação.

$\mathrm{O}$ aumento da experiência tem demonstrado que esses a ressecção hepática por vídeo têm sido ampliados. Grandes ressecções como hepatectomias direita e esquerda ${ }^{(8,9,16)}$ têm sido cada vez mais indicadas. Mais recentemente, até a lobectomia esquerda (bi-segmentectomia II-III) para doador vivo de fígado já tem sido empregada ${ }^{(7)}$. Alguns Serviços como o de O'ROURKE $\mathrm{VL}$ de forma rotineira.

\section{CONCLUSÕES}

A hepatectomia por VL é factível e segura, no entanto, tem indicação para casos selecionados. É, sem dúvida nenhuma, muito bem indicada para pacientes jovens com tumores benignos (principalmente o adenoma hepático) localizados nos segmentos hepáticos II, III, IVb, V e VI. O uso da via laparoscópica não deve porém, modificar as indicações para a ressecção hepática.

A ressecção por VL de hepatocarcinoma periférico em pacientes cirróticos, tem resultados encorajadores pois, aparentemente, tem menor incidência de complicações pósoperatórias. A ressecção de metástases hepáticas, no entanto, ainda necessita de maior experiência. Faltam estudos para comprovar se a ressecção por VL é superior à convencional e, num futuro próximo, devem-se realizar trabalhos comparativos com resultados oncológicos a longo prazo, avaliação dos custos e outras variáveis.

A ressecção hepática por VL é hoje uma realidade e deve fazer parte do armamentário terapêutico do cirurgião de fígado. problemas citados são passíveis de resolução e os limites para e FIELDING ${ }^{(28)}$ na Austrália, realizam hepatectomias direita por 
D’Albuquerque LAC, Herman P. Laparoscopic liver resection. Is it a reality? Arq Gastroenterol. 2006;43(3):243-6.

ABSTRACT - Background - Despite the advances during the last years, liver resection remains as one of the last frontiers in laparoscopic surgery. Published data include case reports and evaluations of small series of patients and are seen with skepticism by many surgeons. Laparoscopic liver resection is a long and hazzardous and long procedure and experience in liver and laparoscopic surgery and an adequate equipment are needed. A critical analysis of the indications, technical aspects and the method was performed. Conclusions - We can conclude that laparoscopic liver resection is safe, feasible and can be considered as an excellent choice for selected cases. There is no doubt that videolaparoscopic liver resection is a reality and should be part of liver surgeon's therapeutic armamentarium.

HEADINGS - Hepatectomy. Laparoscopy.

\section{REFERÊNCIAS BIBLIOGRÁFICAS}

1. Arcarli K. Controversies in the laparoscopic treatment of hepatic hydatic disease. HPB 2004;6:213-21

2. Azagra JS, Gowergen M, Gilbart E, Jacobs D. Laparoscopic anatomical left latera segmentectomy - technical aspects. Surg Endosc. 1996;10:758-61.

3. Belli G, Fantini C, D'Agostino A, Belli A, Russolillo N. Laparoscopic liver resection for hepatocellular carcinoma in cirrhotic patients. HPB. 2004;6:236-46.

4. Buell JF, Thomas MJ, Gersin KS, Merchen TD, Gupta M, Rudish SM, Woodle ES. An initial experience and evolution of laparoscopic hepatic resectional surgery. Surgery. 2004; $136: 804-11$

5. Buell JF, Koffron AJ, Thomas MJ, Rudish S, Abecassis M, Woodle ES. Laparoscopic liver resection. J Am Coll Surg. 2005;200:472-80.

6. Cherqui D, Husson E, Hammoud R, Stéphan F, Bensaid S, Rotman N, Fagniez PL. Laparoscopic liver resections: a feasibility study in 30 patients. Ann Surg. 2000;232:753-62.

7. Cherqui D, Soubrane O, Husson E, Barshasz E, Vignaux O, Ghimouz M, Branchereau S, Chardot C, Gauthier F, Fagniez PL, Houssin D. Laparoscopic living donor hepatectomy for liver transplantation in children. Lancet. 2002;359:392-6.

8. Cherqui D. Laparoscopic liver resection. Br J Surg. 2003;90:644-6.

9. Descottes B, Glineur D, Lachachi F, Valleix D, Paineau J, Hamy A, Morino M, Bismuth H, Castaing D, Savier E, Honore P, Detry O, Legrand M, Azagra JS, Goergen M, Ceuterick M, Marescaux J, Mutter D, de Hemptinne B, Troisi R, Weerts J, Dallemagn B, Jehaes C, Gelin M, Donckier V, Aerts R, Topal B, Bertrand C, Mansvelt B, Van Krunckelsven L, Herman D, Kint M, Totte E, Schockmel R, Gigot JF. Laparoscopic liver resection of benign liver tumors. Results of a multicenter European experience. Surg Endosc. 2003;17:23-30.

10. Elias D, Cavalcanti A, Sabourin JC, Pignon JP, Ducreux M, Lasser P. Results of 136 curative hepatectomies with a safety margin of less than $10 \mathrm{~mm}$ for colorectal metastases. J Surg Oncol. 1998;69:88-93.

11. Farges O, Jagot P, Kirstetter P, Marty J, Belghiti J. Prospective assessment of the safety and benefit of laparoscopic resections. J Hepatobiliary Pancreat Surg. 2002;9:242-8.

12. Ferzli G, David A, Kiel T. Laparoscopic resection of a large hepatic tumor. Surg Endosc. 1995;9:733-5.

13. Fong Y, Jarnagin W, Conlon KC, DeMatteo R, Dougherty E, Blumgart LH. Handassisted laparoscopic liver resection. Arch Surg. 2000;135:854-9.

14. Gagner M, Rogula T, Selzer D. Laparoscopic liver resection: benefits and controversies. Surg Clin North Am. 2004;84:451-62.

15. Gigot JF, Glineur D, Azagra JS, Goergen M, Centerick M, Morino M. Laparoscopic liver resection for malignant liver tumors: preliminary results of a multicenter European study. Ann Surg. 2002;236:90-7.

16. Huscher CG, Lirici MM, Chiodini S. Laparoscopic liver resections. Semin Laparosc Surg. 1998;5:204-10

17. Inagaki H, Kurokawa T, Nonami T, Sakamoto J. Hand-assisted laparoscopic left latera segmentectomy of the liver for hepatocellular carcinoma with cirrhosis. J Hepatobiliary Pancreat Surg. 2003;10:295-8.
18. Kaneko H, Takagi S, Otsuka Y, Tsuchiya M, Tamura T, Maeda T, Shiba T. Laparoscopic liver resection of hepatocellular carcinoma. Am J Surg. 2005;189:190-4

9. Katkhouda N, Mavor E. Laparoscopic management of benign liver disease. Surg Clin North Am. 2000;80:1203-11.

20. Ker CG, Chen HY, Juan CC, Chang WS, Tsai CY, Lo HW, Yau MT. Laparoscopic subsegmentectomy for hepatocellular carcinoma with cirrhosis. Hepatogastroenterology. 2000;47:1260-3.

21. Lacy AM, Garcia-Valdecasas JC, Delgado S, Castells A, Taura P, Pique JM. Laparoscopicassisted colectomy versus open colectomy treatment of non-metastatic colon cancer: a randomized trial. Lancet. 2002;359:2224-9.

22. Laurent A, Cherqui D, Lesurtel M, Brunetti F, Tayar C, Fagniez PL. Laparoscopic liver resection for subcapsular hepatocellular carcinoma complicating chronic liver disease. Arch Surg. 2003;138:763-9.

23. Lesurtel M, Cherqui D, Laurent A, Tayar C, Fagniez PL. Laparoscopic versus open left lateral hepatic lobectomy: a case control study. J Am Coll Surg. 2003;196:236-42.

24. Linden BC, Humar A, Sielaff TD. Laparoscopic stapled left lateral segment liver resection - technique and results. J. Gastrointest Surg. 2003;7:777-82.

25. Machado MAC, Herman P, Makdissi FF, Galvão FH. Ressecções hepáticas por videolaparoscopia: utilidade da técnica de hemi-Pringle. Rev Bras Videocir. 2005;3:59.

26. Machado MA, Makdissi FF, Bacchella T, Machado MC. Hemihepatic ischemia for laparoscopic liver resection. Surg Laparosc Endosc Percutan Tech. 2005;15:180-3.

27. O'Rourke N, Shaw I, Nathanson L, Martin I, Fielding G. Laparoscopic resection of hepatic colorectal metastases. HPB. 2004;6:230-5.

28. O'Rourke N, Fielding G. Laparoscopic right hepatectomy: surgical technique. J Gastrointest Surg. 2004;8:213-6.

29. Poon RT, Fan ST, NG IO, Wong J. Significance of resection margin in hepatectomy for hepatocellular carcinoma: a critical reappraisal. Ann Surg. 2000;231:544-51.

30. Poulin EC, Mamazza J, Schlachta CM, Gregoire R, Roy N. Laparoscopic resection does not adversely affect early survival curves in patients undergoing surgery for colorectal adenocarcinoma. Ann Surg. 1995;229:487-92.

31. Rau HG, Buttler E, Meyer G, Schardley HM, Schildberg FW. Laparoscopic liver resection compared with conventional partial hepatectomy: a prospective analysis. Hepatogastroenterology. 1998;45:2333-8.

32. Soper NJ, Brunt LM, Kerbl K. Laparoscopic general surgery. N Engl J Med. 1994;330:409-19.

33. Vibert E, Kouider A, Gayet B. Laparoscopic anatomic liver resection. HPB. 2004;6:222-9.

34. Vittimberga FJ, Foley DP, Meyers WC, Callery MP. Laparoscopic surgery and the systemic immune response. Ann Surg. 1998;227:226-34. 